\title{
A esteganografia poderia ser utilizada para aumentar o engajamento dos estudantes de engenharia?
}

Isabella de Souza Couto, Universidade de Pernambuco, isc @ poli.br Felipe Alberto Barbosa Simão Ferreira, Universidade Federal de Pernambuco, felipe.bsferreira@ufpe.br

Francisco Madeiro, Universidade de Pernambuco, madeiro@poli.br

Resumo. Nos cursos de engenharia do Brasil mais da metade dos estudantes não chegam a concluir a graduação. $O$ alto índice de evasão ocorre devido a diversos fatores, como, por exemplo, a metodologia de ensino adotada pelos professores, as sucessivas reprovações nas disciplinas do ciclo básico e a falta de relação entre as disciplinas do ciclo básico e profissional. Levando em consideração essa problemática, este trabalho aborda o desenvolvimento de duas apresentações, para que sejam utilizadas como aula, acompanhadas de dinâmicas, para apresentar aos alunos iniciantes do curso de Engenharia Elétrica de Telecomunicações uma das possíveis áreas de atuação acadêmica e profissional, a segurança da informação, mais precisamente a esteganografia. Para uma das aulas foi desenvolvido um aplicativo, chamado ImEstegApp, que teve por finalidade mostrar de forma prática aos alunos uma das técnicas de esteganografia digital. A abordagem proposta proporcionou um aumento tanto no grau de engajamento como no de motivação dos discentes.

Palavras-chave: evasão, engenharia, esteganografia, engajamento, motivação dos alunos.

\section{Could steganography be used to increase the engagement of engineering students?}

Abstract. In engineering courses available in Brazil, more than a half of the students do not finish their degree. This high dropout rate is due to a number of factors, such as the teaching methodology, successive failures in the basic cycle subjects and lack of relationship between subjects of basic and professional cycles. Taking into account the teaching methodology factor, the main objective of this work is to develop two presentations to be used as classes. They are accompanied by a set of activities in order to introduce to the beginning students of the Telecommunications Engineering undergraduation course one of the possible academic and professional areas they will be capable to work with. The classes are focused on information security, more precisely steganography. An application, called ImEstegApp, was developed with the purpose of showing students one of the techniques of digital steganography in a practical way. The proposed approach provided an increase in the engagement and motivation degree of students.

Keywords: dropout, engineering, steganography, engagement, student's motivation.

\section{INTRODUÇÃO}

Um dos maiores problemas enfrentados pelas instituições de ensino superior dos cursos de Engenharia é a evasão dos estudantes. Com base em dados do Ministério da Educação (MEC), a Confederação Nacional da Indústria (CNI) constatou que menos de $50 \%$ dos estudantes de engenharia concluem a graduação no país (Gomez et al., 2015). O baixo índice de graduados no curso implica um déficit de mão de obra necessária para atender as demandas do país, bem como se torna um mau indicador para as instituições. 
Vários fatores podem ocasionar o abandono dos cursos de engenharia. Uma análise realizada por Almeida e Godoy (2016) apontou que as principais causas são de origem pedagógica, sendo algumas delas as sucessivas reprovações nas disciplinas do ciclo básico, a metodologia de ensino utilizada pelos professores, a ausência de relacionar as disciplinas do ciclo básico com o profissional, além da falta de atividades práticas. Um fator relevante para a retenção de alunos em cursos de nível superior é a motivação do estudante (Paura e Arhipova, 2014). Um estudo sobre a evasão e retenção de alunos de graduação realizado em uma universidade federal brasileira (da Costa et al., 2018) aponta que ações motivacionais são importantes para manter a retenção dos alunos no curso. Além de sua importância para diminuir as taxas de evasão, a motivação dos alunos é fundamental para um bom desempenho com relação ao processo de aprendizagem (do Canto Filho et al., 2012). Devido a sua importância, políticas pedagógicas vêm sido desenvolvidas com base na análise motivacional dos estudantes como forma de aumentar a retenção (Toledo et al., 2019). Um exemplo de trabalho voltado para motivação incluiu o uso de realidade aumentada no processo de ensino para aumentar a motivação dos estudantes em cursos de engenharia (Kaur et al., 2020).

Diante desses fatores, este trabalho tem como objetivo apresentar o desenvolvimento de dois experimentos que visaram aumentar o engajamento e motivação dos estudantes do curso de Engenharia Elétrica de Telecomunicações da Universidade de Pernambuco ao apresentar aos mesmos uma vertente da área segurança da informação que só seria abordada nas aulas do ciclo profissional, mas que se reputa como potencialmente capaz de gerar motivação e, assim, contribuir para diminuir a evasão.

Para tanto, desenvolveram-se duas apresentações, acompanhadas de um conjunto de atividades, para apresentar aos alunos uma das possíveis áreas de atuação, tanto acadêmica quanto profissional: a segurança da informação, mais precisamente a esteganografia. O material e um novo aplicativo desenvolvidos foram apresentados para uma turma de Introdução a Engenharia, com a finalidade de aumentar o interesse dos mesmos pelo curso e, consequentemente, diminuir a evasão.

O presente trabalho encontra-se organizado como segue. A seção 2 aborda a esteganografia desde a antiguidade até os dias atuais. A seção 3 apresenta a metodologia do trabalho. Na seção 4 a ferramenta desenvolvida é descrita. As seções 5 e 6 estão destinadas aos resultados e conclusão, respectivamente.

\section{ESTEGANOGRAFIA}

Esteganografia é uma palavra derivada do grego: estegano significa esconder e grafia significa escrita. Logo, esteganografia significa escrita encoberta. A técnica consiste em camuflar as informações com o objetivo de que as mesmas passem despercebidas e, consequentemente, não sejam detectadas por indivíduos não autorizados (Kadhim et al., 2019; Denemark et al., 2018; Cox et al., 2007).

A arte de esconder informações é uma técnica milenar que começou a ser utilizada na Grécia Antiga pela necessidade de os reinos se comunicarem de forma secreta em tempos de guerra. Os primeiros registros da utilização do método foram descritos no texto Histórias, de Heródoto, pai da História, e datam do século V a.C. Um dos relatos narra que um grego chamado Histio desejava enviar uma mensagem sigilosa para o seu superior, Aristágoras. Para isso utilizou como mensageiro o seu escravo mais fiel, raspou sua cabeça para que assim pudesse escrever a mensagem secreta em seu couro cabeludo e aguardou que o cabelo crescesse para o enviar a Aristágoras. A informação 
secreta solicitava que o superior iniciasse uma revolta contra os persas o mais rápido possível (Tkotz, 2005; Cox et al., 2007).

Outro método de esteganografia são as tintas invisíveis. Seus primeiros relatos datam do século I d.C. quando o poeta romano Plínio, o Velho, usou o leite de uma planta chamada thithymallus para escrever mensagens secretas que poderiam ser reveladas quando aquecido o papel (Tkotz, 2005). As tintas invisíveis também foram muito usadas nas duas grandes guerras mundiais. Os espiões as utilizavam para ocultar informações nos mais diversos meios, como livros, jornais e revistas (Julio et al., 2007).

A técnica de micropontos e cifras nulas foram utilizadas durante a Segunda Guerra Mundial. A primeira consiste em tirar uma foto da mensagem secreta e posteriormente reduzi-la ao tamanho de um ponto final e assim incorporá-la nos textos. Já as cifras nulas consistem em produzir um texto, aparentemente inocente, de tal maneira que, ao juntar as primeiras letras de cada palavra do texto se obtinha a informação secreta (Julio et al., 2007; Cheddad et al., 2010).

A esteganografia passou a ser digital devido aos avanços tecnológicos e surgimento da Internet. A esteganografia digital consiste em esconder dados que podem ser, por exemplo, imagens, vídeos, textos, dentro de algum objeto digital, que analogamente aos dados pode ser, por exemplo, uma imagem, vídeo, texto (Artz, 2001; Hussain et al., 2018). Ela possui várias aplicações como comunicação segura de informações confidenciais dentro de empresas e comunicação militar (Ansari et al., 2019; Subhedar e Mankar, 2014). Por ser uma técnica que, em geral, não levanta suspeitas, pode também ser utilizada no cybercrime por hackers ou terroristas para divulgar dados sigilosos que foram roubados (Cheddad et al., 2010).

$\mathrm{Na}$ esteganografia digital alguns termos são utilizados. O chamado dado embutido é a informação que se deseja enviar de maneira secreta. Pode ser texto, imagem, voz, áudio, por exemplo. Esta informação é escondida no objeto de cobertura, que também pode ser imagem, voz, vídeo, texto, entre outros. Objeto de cobertura é um termo geral. Quando se trata de um meio específico, o nome é alterado de acordo com o meio, por exemplo, é chamado de imagem de cobertura ao se utilizar uma imagem para esconder a mensagem (Rocha et al., 2003; Hussain et al., 2018). Após o processo de incorporar o dado embutido no objeto de cobertura se obtém o estego-objeto.

Para o presente trabalho foi abordada a esteganografia em imagens digitais. Uma das técnicas utilizadas para ocultação de informação em imagens é a inserção no bit menos significativo (LSB - Least Significant Bit) (Rocha et al., 2003). A técnica consiste em utilizar os bits menos significativos dos pixels para inserir as informações sigilosas (Hussain et al., 2018). É válido ressaltar que, uma vez escolhida adequadamente a imagem de cobertura, uma alteração nos LSBs não causa mudanças perceptíveis ao olho humano. No caso das imagens monocromáticas PGM (Portable Gray Map) com 256 níveis de cinza, cada pixel é representado por 8 bits.

Cumpre mencionar que a esteganálise tem como objetivo identificar se o estegoobjeto possui mensagem oculta (Rashid et al., 2016; Ansari et al., 2019). Para verificar a existência de informações ocultas o esteganalista pode aplicar ataques nas imagens. Um deles é o ataque visual, que consiste em analisar a olho nu o plano de bit LSB da imagem. De maneira geral uma imagem natural na qual não foi inserida informação apresenta um padrão "aleatório" no plano de bit LSB. Já quando a imagem esconde algo, em vez desse padrão "aleatório", a depender da técnica de esteganografia, pode-se observar um rastro na região de imagem na qual a mensagem foi inserida. Essa diferença pode ser verificada na Figura 1. 


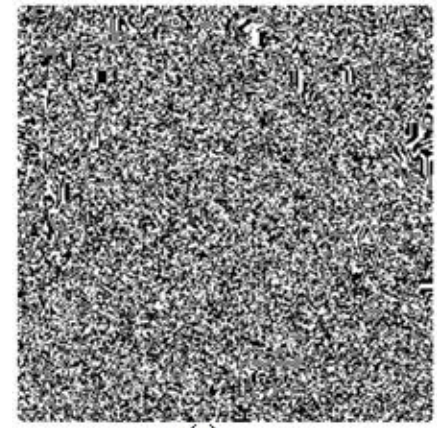

(a)

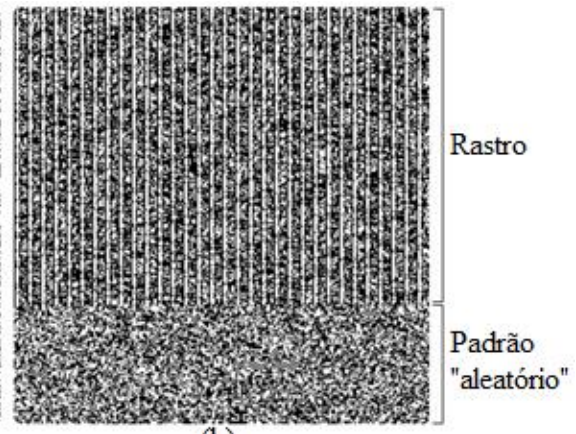

(b)

Figura 1 - Ilustração de um ataque visual: (a) plano de bit LSB sem informação oculta, (b) plano de bit LSB com informação oculta.

Fonte: Autores.

\section{MATERIAIS E MÉTODOS}

Para realizar os experimentos foram aplicadas duas aulas em uma turma de Introdução à Engenharia, uma disciplina de primeiro período, com 18 estudantes do curso de Engenharia Elétrica de Telecomunicações, na Universidade de Pernambuco. $\mathrm{Na}$ primeira aula, foi abordada a esteganografia na antiguidade, e na segunda, foi abordada a esteganografia em imagens digitais. Antes de abordar o conteúdo da primeira aula foi solicitado que os alunos respondessem uma pesquisa de opinião.

Após a pesquisa de opinião com participantes não identificados, deu-se início a abordagem do conteúdo. Primeiramente foi apresentado aos discentes o significado de esteganografia e feita uma comparação entre esta técnica e a técnica de criptografia, depois se deu a apresentação dos aspectos históricos da ocultação de informação. Ao abordar as tintas invisíveis, foi proposta uma dinâmica demonstrativa para que algum estudante pudesse observar uma frase sendo revelada. Para isso foi utilizada uma folha de jornal como meio de cobertura, a mensagem foi escrita com uma tinta de amido de milho e para revelar o aluno deveria passar uma mistura de 20 gotas de solução de iodo misturada a $5 \mathrm{ml}$ de água. $\mathrm{O}$ intuito da dinâmica foi que o aluno pudesse experimentar a sensação de revelar uma mensagem oculta.

Ao fim da aula de conteúdo foi proposta uma dinâmica com uma técnica de criptografia, a cifra de César, em conjunto com a técnica de tinta invisível de amido de milho. A cifra de César é uma cifra de substituição monoalfabética, a qual consiste em substituir cada letra de um texto por outra letra, do mesmo alfabeto, porém deslocado. Assim, por exemplo, considerando um deslocamento de três letras adiante, a palavra "casa" é cifrada com "fdvd".

Os 14 alunos presentes em sala foram divididos em dois grupos de quatro integrantes e dois grupos de três integrantes. A cada grupo foi entregue uma folha de jornal que continha aparentemente apenas a matéria impressa, porém cada folha possuía uma mensagem que foi ocultada utilizando uma tinta invisível feita com amido de milho e água, e a mensagem, por sua vez, estava criptografada com cifra de César. O intuito da dinâmica era que cada grupo tentasse desvendar qual era a mensagem contida no jornal. A dinâmica parece ter instigado os alunos a colocar em prática o que haviam aprendido.

$\mathrm{Na}$ segunda aula foi abordada a esteganografia em imagens digitais. Primeiramente foram apresentados os motivos para a esteganografia ter se tornado digital, bem como exemplos de utilização de aplicação da técnica. Foram apresentados a terminologia da área e os fundamentos básicos de representação binária e de imagens no formato PGM. Então foi apresentada a técnica de inserção no LSB, bem como a esteganálise no âmbito de ataque visual. 
A dinâmica realizada nesta aula consistiu em duas atividades práticas. A primeira possuía duas perguntas para verificar o aprendizado sobre representação binária e LSB, respondidas individualmente pelos 14 alunos. A segunda atividade consistiu em três perguntas técnicas sobre a esteganografia LSB. Para responder, os estudantes foram introduzidos ao ImEstegApp, aplicativo desenvolvido para ser usado na aula. Apresentado o aplicativo, os alunos responderam perguntas sobre qualidade de estegoimagem por meio de PSNR (Peak Signal-to-Noise Ratio) e sobre ataque visual.

Ao final da segunda aula foi solicitado que os alunos respondessem outra pesquisa de opinião para determinar se o tema lhes gerou interesse, se o aplicativo móvel foi útil para fixar o conteúdo, além de responder qual o grau de engajamento e motivação após as dinâmicas.

\section{FERRAMENTA DESENVOLVIDA}

O aplicativo, chamado ImEstegApp, foi desenvolvido para aparelhos móveis que utilizam o sistema Android, sendo também possível acessá-lo por navegador da web.

A ferramenta foi desenvolvida para ser utilizada pelos alunos que participaram da segunda aula. Ao utilizá-la eles puderam reforçar não apenas o conhecimento teórico sobre o conteúdo abordado, mas também vivenciar a ocultação de uma mensagem em uma imagem. Exemplos do uso de ferramentas computacionais para auxiliar estudantes na melhor compreensão dos mais diversos conteúdos podem ser vistos em (Karnavas e Lygouras, 2018; Biasi et al., 2019; Feijó et al., 2019; Panegalli et al., 2019).

A parte visual do ImEstegApp foi desenvolvida utilizando a linguagem HTML5 e para fazer as funcionalidades disponíveis no aplicativo foi utilizada a linguagem de programação JavaScript. O mesmo foi elaborado para ser intuitivo e de fácil utilização. A Figura 2 ilustra a tela inicial e a função de escolha da imagem de cobertura e a Figura 3 ilustra as funções de inserção e extração de texto.

No aplicativo é possível utilizar todos os planos de bit da imagem, do LSB até o MSB (Most Significant Bit), para ocultação da mensagem nas imagens. O primeiro passo, ilustrado na Figura 2(b), consiste em escolher a imagem na qual se quer ocultar o texto, a imagem de cobertura. O aplicativo dispõe de nove imagens monocromáticas de tamanho 256x256, mas também é possível que o usuário escolha uma imagem disponível nos arquivos do próprio aparelho, o ideal é que ela tenha as mesmas dimensões das imagens disponíveis no aplicativo, caso contrário a imagem aparecerá cortada. Na função de visualizar o plano de bit o usuário pode ver a imagem no plano de bit LSB até o MSB. Após a escolha da imagem, o usuário deve inserir a mensagem que se deseja esconder, conforme apresentado na Figura 3(a). Para fins didáticos, o aplicativo dispõe de uma mensagem padrão, caso o usuário não queira digitar sua própria mensagem. Esta mensagem padrão foi retirada do artigo Steganography do Wikipedia através do link https://en.wikipedia.org/wiki/Steganography. É possível nesse passo escolher em qual plano de bit se deseja inserir a mensagem e, após a inserção, o usuário pode visualizar a imagem original e a estego-imagem. Apesar de $o$ aplicativo dispor de todos os planos de bit da imagem para inserir a mensagem desejada é recomendado que se use o LSB para ocultar a informação. $\mathrm{O}$ aplicativo possui outros planos de bit para fins didáticos, pois assim o estudante pode observar a diferença entre a imagem de cobertura e a estego-imagem. O usuário também pode salvar em seu dispositivo a estego-imagem gerada, para posteriormente poder envia-la a algum amigo, que, por sua vez, poderá usar o aplicativo para descobrir a mensagem secreta. Depois de inserida a mensagem o usuário pode extrair o texto, ilustrado na Figura 3(b), e neste passo ele deverá escolher de qual plano de bit ele deseja extrair o texto. Se o plano de 
bit escolhido for aquele em que a mensagem foi inserida, o texto que foi inserido aparecerá na caixa "texto extraído", mas caso o usuário escolha algum dos planos de bit que não foi utilizado para a ocultação do texto, aparecerão vários símbolos aleatórios. Além de todas essas funcionalidades o usuário também pode realizar o ataque visual na estego-imagem gerada, o qual foi ilustrado na Figura 1.

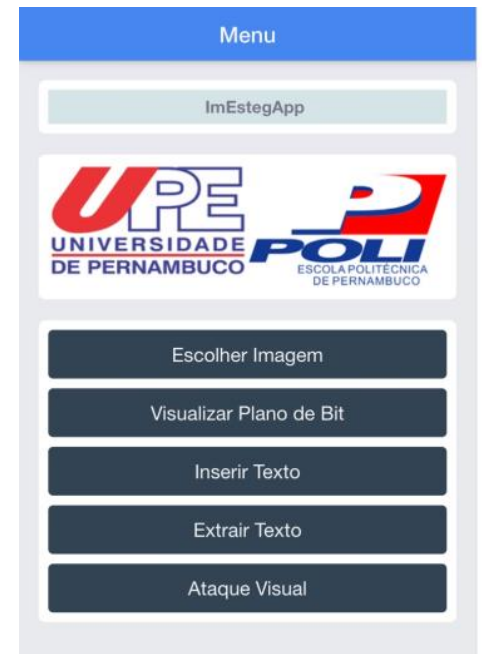

(a)

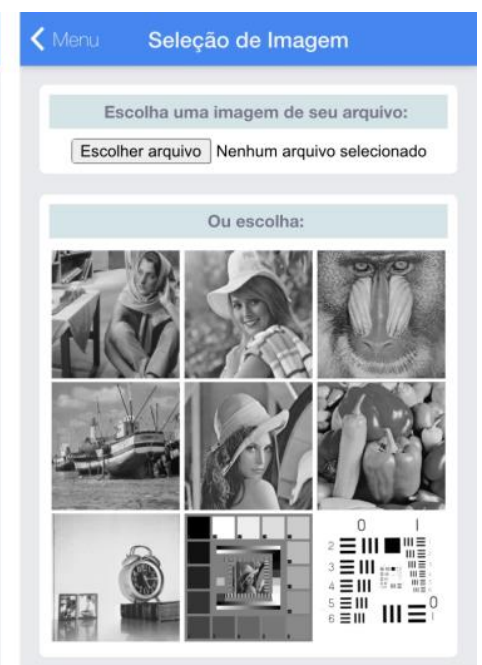

(b)

Figura 2 - ImEstegApp: (a) tela inicial e (b) tela para escolha da imagem de cobertura. Fonte: Autores.

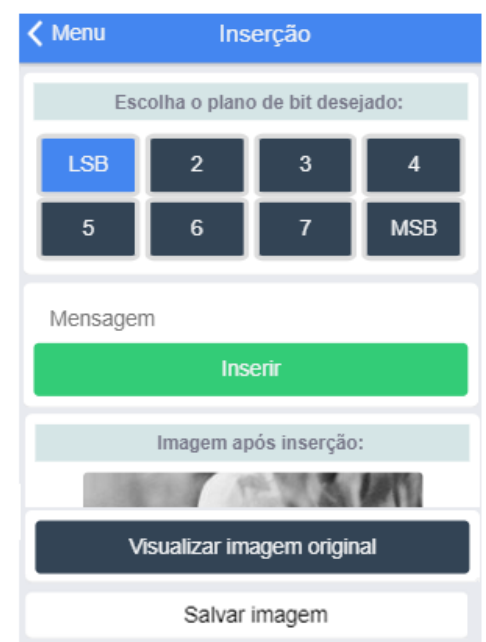

(a)

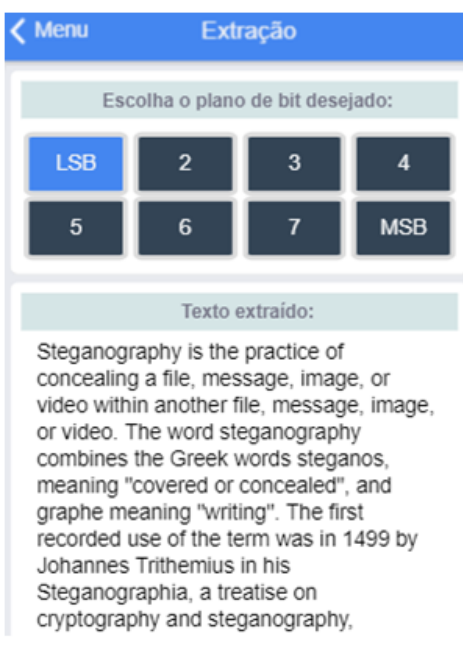

(b)

Figura 3 - Telas do ImEstegApp: (a) inserção de texto e (b) extração de texto. Fonte: Autores.

\section{RESULTADOS}

As pesquisas de opinião solicitadas no começo da primeira e no fim da segunda aula foram elaboradas no Formulário Google e disponibilizadas para os alunos através de um $Q R$ Code. Respondidas de forma anônima, elas tiveram o objetivo de gerar resultados acerca do experimento proposto a fim de verificar a sua contribuição. A turma de Introdução à Engenharia do curso de Engenharia Elétrica de Telecomunicações da Universidade de Pernambuco tem 18 alunos e em ambas as aulas 14 alunos estiveram presentes. 
As Figuras 4 e 5 apresentam gráficos com respostas para as perguntas realizadas na primeira e segunda pesquisa de opinião, respectivamente. O título de cada gráfico corresponde a cada uma das perguntas.

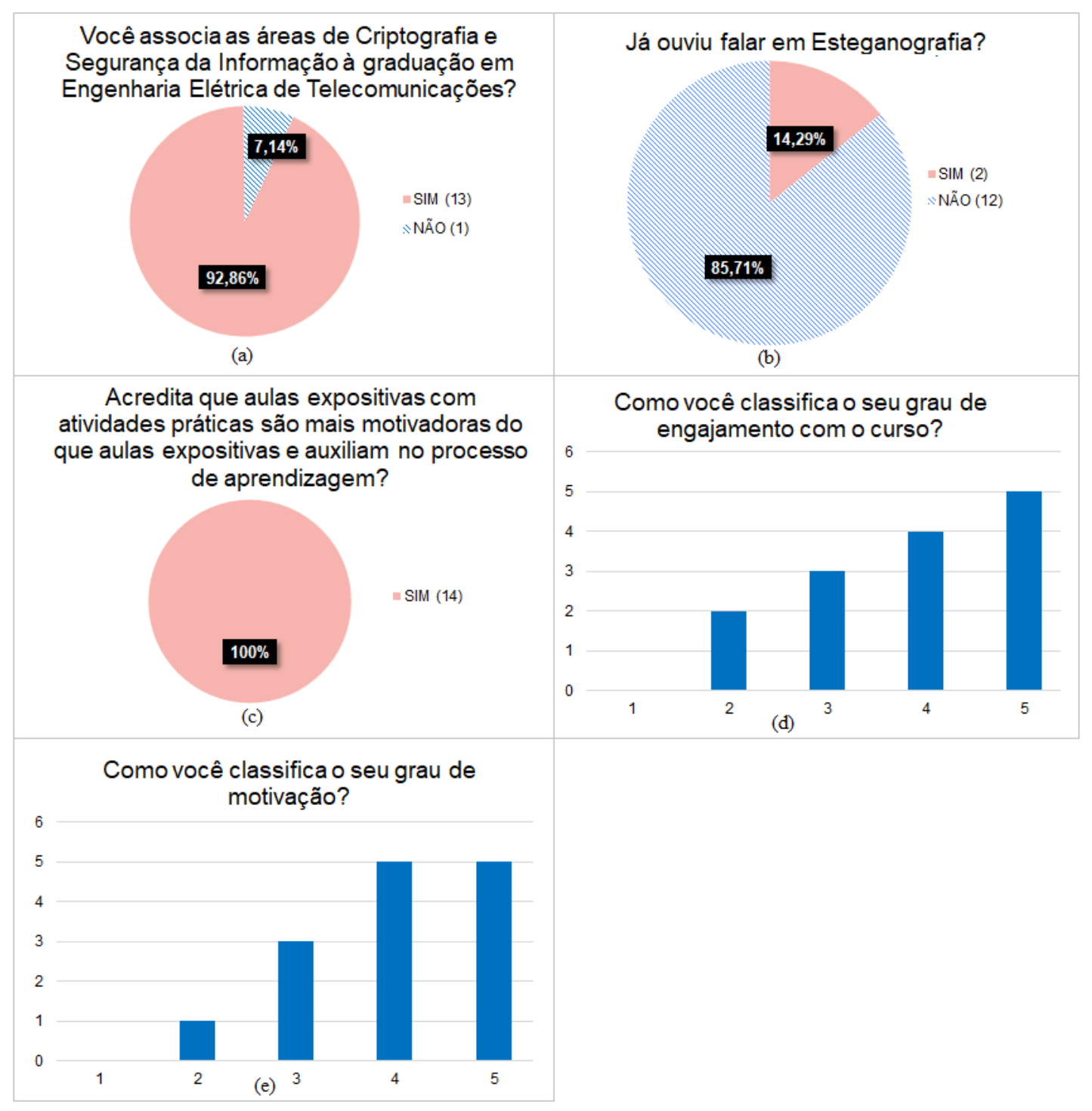

Figura 4 - Respostas das perguntas da primeira pesquisa de opinião. Fonte: Autores.

$\mathrm{Na}$ Figura 4(a), observa-se que 13 dos 14 alunos responderam que associam as áreas de Criptografia e Segurança da Informação ao curso. Mesmo o tema apresentado tendo relação com a Criptografia e Segurança da Informação ele era desconhecido por $85,71 \%$ dos estudantes da turma, como mostrado na Figura 4(b), e despertou interesse em $92,86 \%$ dos mesmos, Figura 5(a).

Todos os discentes presentes nas aulas concordaram que aulas teóricas seguidas de atividades práticas motivam e auxiliam no processo de aprendizagem, como mostrado na Figura 4(c). Responderam "Sim" quando foram questionados se as aulas, nas quais foi abordado o conteúdo, e, em seguida realizadas dinâmicas (utilizando tintas invisíveis e o ImEstegApp), auxiliaram para a fixação do conteúdo, Figura 5(c). Apenas um dos 14 alunos, equivalente a 7,14\%, como ilustrado na Figura 5(b), não concordou ter sido interessante abordar a história antiga da esteganografia antes da esteganografia na 
atualidade. $\mathrm{O}$ resultado se mostrou positivo e confirmou que a maioria dos estudantes julga importante conhecer a história do conteúdo.

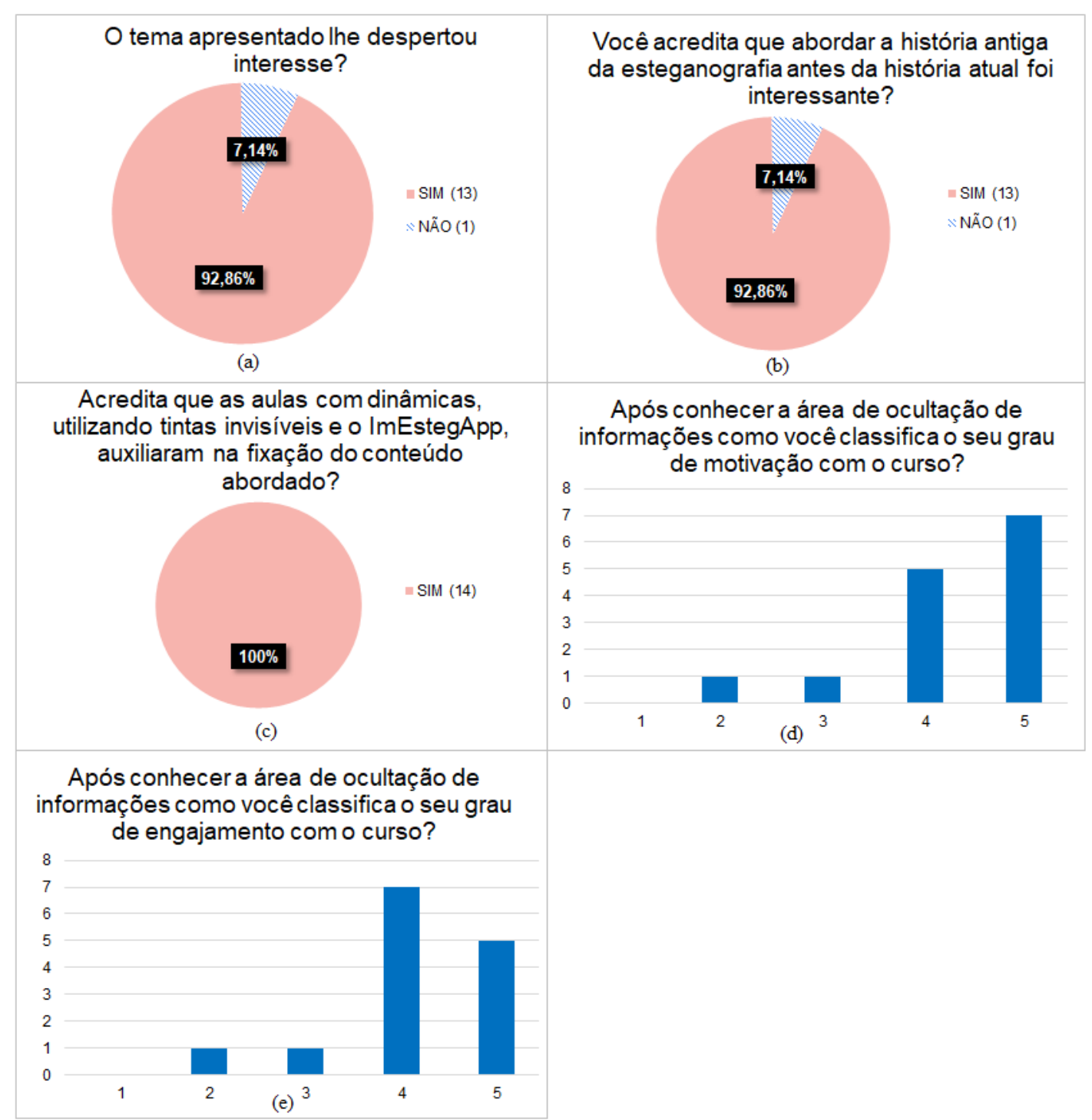

Figura 5 - Respostas da segunda pesquisa de opinião. Fonte: Autores.

Para verificar o grau de engajamento e motivação dos alunos antes e após as aulas da abordagem proposta, foi feita a mesma pergunta nas duas pesquisas de opinião. Em ambas os alunos deveriam dar uma nota para classificar o seu engajamento e motivação, numa escala de números naturais de 1 (totalmente não engajado/motivado) a 5 (totalmente engajado/motivado). Na primeira pesquisa de opinião a média do engajamento da turma foi de aproximadamente 3,86, como ilustrado na Figura 4(d), já na segunda pesquisa essa média subiu para 4,14, como mostrado na Figura 5(e). Já no quesito da motivação a média antes da aplicação das aulas era de 4,00, como apresentado na Figura 4(e), e após a aplicação das aulas propostas passou para 4,29, como ilustrado na Figura 5(d). Esses resultados se mostram positivos e sugerem que quanto mais cedo os discentes tiverem contato com conteúdo juntamente com dinâmicas voltados para as disciplinas do ciclo profissional mais eles se sentem motivados com o curso. E com isso, é possível que o índice de evasão do curso diminua. 


\section{CONSIDERAÇÕES FINAIS}

O índice de evasão dos estudantes de Engenharia é um problema com o qual as instituições de ensino superior têm que lidar. A literatura mostra que dentre as causas que podem levar ao alto índice, destacam-se as de origem pedagógica. Diante desse cenário, o presente trabalho objetivou desenvolver duas aulas para apresentar, aos estudantes iniciantes do curso de Engenharia Elétrica de Telecomunicações da Universidade de Pernambuco, uma de suas áreas de atuação. O objetivo do trabalho foi apresentar aos estudantes o conteúdo esteganografia, o qual é abordado apenas durante o ciclo profissional, para aumentar o engajamento e a motivação dos estudantes, para que assim a taxa de evasão possa reduzir.

Ao analisar os resultados obtidos de duas pesquisas de opinião foi possível verificar que, após as duas aulas aplicadas, 13 dos 14 alunos presentes da turma informaram que se interessaram pelo conteúdo apresentado, e todos acreditam que aulas expositivas acompanhadas de atividades práticas são mais motivadoras que aulas expositivas e auxiliam no processo de aprendizagem. Além disso, comparando as duas pesquisas de opinião, tanto o grau de engajamento quanto o de motivação apresentaram um aumento. Assim, apresentar conteúdos diretamente relacionados ao curso adotando atividades práticas que abordam a história de uma determinada área pode contribuir para aumentar o engajamento e a motivação dos discentes.

O modelo das aulas aqui mostrado pode continuar sendo apresentado para as próximas turmas da disciplina considerada. Atividades semelhantes às descritas neste trabalho podem ser criadas e aplicadas a outras disciplinas do curso. Como opção de trabalhos futuros, outras técnicas de esteganografia, envolvendo imagem, vídeo ou áudio, podem ser incorporadas ao aplicativo.

\section{REFERÊNCIAS BIBLIOGRÁFICAS}

ALMEIDA, E.; GODOY, E. V. A evasão nos cursos de engenharia: Uma análise a partir do COBENGE. In: XLIV Congresso Brasileiro de Educação em Engenharia (COBENGE), 2016, Natal. Anais, 2016, v. Único, p. 1-10.

ANSARI, A. S.; MOHAMMADI, M. S.; PARVEZ, M. T. A comparative study of recent steganography techniques for multiple image formats. International Journal of Computer Network and Information Security, v. 11, n. 1, p. 11-25, 2019.

ARTZ, D. Digital steganography: hiding data within data. IEEE Internet Computing, v. 5, n. 3, p. 75-80, 2001.

BIASI, M. R. Di; VALENCIA, G. E.; OBREGON, L. G. A new educational thermodynamic software to promote critical thinking in youth engineering students. Sustainability, v. 12, n. 1, p. 1-17, 2019.

CHEDDAD, A.; CONDELL, J.; CURRAN, K.; Mc KEVITT, P. Digital image steganography: Survey and analysis of current methods. Signal Processing, v. 90, n. 3, p. 727-752, 2010.

COX, I.; MILlER, M.; BLOOM, J.; FRIDRICH, J.; KALKER, T. Digital Watermarking and Steganography. Morgan Kaufmann. 2007. 624 p.

DA COSTA, F. J.; BISPO, M. S.; PEREIRA, R. C. F. Dropout and retention of undergraduate students in management: a study at a Brazilian Federal University. RAUSP Management Journal, v. 53, n. 1, p. 74-85, 2018.

DENEMARK, T.; BAS, P.; FRIDRICH, J. Natural steganography in JPEG compressed images. Electronic Imaging, v. 2018, p. 316-1-316-10, 2018. 
DO CANTO FILHO, A. B.; FERREIRA, L. F.; BERCHT, M.; TAROUCO, L. M. R.; DE LIMA, J. V. Objetos de aprendizagem no apoio à aprendizagem de engenharia: explorando a motivação extrínseca. RENOTE - Revista Novas Tecnologias na Educação, v. 10, n. 3, 2012.

FEIJÓ, L. P.; DE BARROS FILHO, E. M.; FERREIRA, E. B. C.; AUGUSTO, K. L. Desenvolvimento e avaliação de um aplicativo para apoio ao ensino de psicopatologia. RENOTE - Revista Novas Tecnologias na Educação. v. 17, n. 3, p. 244-252, 2019. GOMEZ, M. F.; REMOR, C. R.; DE MARCO, M. T.; BETZEK, S. B. F. Evasão na engenharia: $\mathrm{O}$ caso dos cursos da UTFPR câmpus Medianeira tendo como acesso o SiSU. Revista Eletrônica Científica Inovação e Tecnologia Universidade Tecnológica Federal do Paraná Câmpus Medianeira, Paraná, 2015. Disponível em: <https://periodicos.utfpr.edu.br/recit/article/view/4294>. Acesso em: 10 nov. 2019. HUSSAIN, M.; WAHAB, A. W. A.; IDRIS, Y. I. B.; HO, A. T.; JUNG, K. H. Image steganography in spatial domain: A survey. Signal Processing: Image Communication, v. 65, p. 46-66, 2018.

JULIO, E. P.; BRAZIL, W. G.; ALBUQUERQUE, C. V. N. Esteganografia e suas Aplicações. In: Luci Pirmes; Flávia Delicato. (Org.). Livro de Minicursos do SBSEG. Rio de Janeiro: Sociedade Brasileira de Computação, 2007, v. 7, p. 54-102.

KADHIM, I. J.; PREMARATNE, P.; VIAL, P. J.; HALLORAN, B. Comprehensive survey of image steganography: Techniques, evaluations, and trends in future research. Neurocomputing, v. 335, p. 299-326, 2019.

KARNAVAS, Y. L.; LYGOURAS, E. I. Synchronous machine analysis and modelling in LabVIEW: An educational tool for transient stability studies. International Journal of Electrical Engineering Education, 2018. Disponível em: < https://journals.sagepub.com/doi/10.1177/0020720918791422>. Acesso em: 7 abr. 2020.

KAUR, D. P.; MANTRI, A.; HORAN, B. Enhancing student motivation with use of augmented reality for interactive learning in engineering education. Procedia Computer Science, v. 172, p. 881-885, 2020.

PANEGALLI, F. S.; BERNARDI, G.; CORDENONSI, A. Z. Super Mario Logic: um jogo sério para auxiliar no processo de ensino e aprendizagem de lógica de programação. RENOTE - Revista Novas Tecnologias na Educação. v. 17, n. 1, p. 244-253, 2019.

PAURA, L.; ARHIPOVA, I. Cause analysis of students' dropout rate in higher education study program. Procedia-Social and Behavioral Sciences, v. 109, p. 12821286, 2014.

RASHID, A.; MISSEN, M.; SALAMAT, N. Analysis of steganography techniques using least significant bit in grayscale images and its extension to colour images.

Journal of Scientific Research \& Reports (JSRR), v. 9, p. 1-14, 2016.

ROCHA, A. R.; COSTA, H. A. X.; CHAVES, L. M. Camaleão: Um Software para Segurança Digital Utilizando Esteganografia. Lavras: Departamento de Ciências da Computação/UFLA, 2003. 108 p. Monografia.

SUBHEDAR, M. S.; MANKAR, V. H. Current status and key issues in image steganography: A survey. Computer Science Review, v. 13, p. 95-113, 2014.

TKOTZ, V. Criptografia: segredos embalados para viagem. São Paulo: Novatec. 2005. $355 \mathrm{p}$.

TOLEDO, B. B; ASTUDILlO, D. F. T.; RUSSO, G.; PARIZOTTO, L. A.; GRIMONI, J. A. B.; NAKAO, O. S. Avaliação da motivação de um aluno de engenharia/Evalution of the motivation of the engineering student. Brazilian Applied Science Review, v. 3, n. 4, p. 1892-1906, 2019. 